\title{
Instrumental profile of MYTHEN detector in Debye-Scherrer geometry
}

\author{
Fabia Gozzo*, I , Antonio Cervellino ${ }^{\mathrm{I}}$, Matteo Leoni ${ }^{\mathrm{II}}$, Paolo ScardiI ${ }^{\mathrm{II}}$, Anna Bergamaschi ${ }^{\mathrm{I}}$ and Bernd Schmitt $^{\mathrm{I}}$ \\ I Paul Scherrer Institut, Swiss Light Source, Villigen PSI, Switzerland

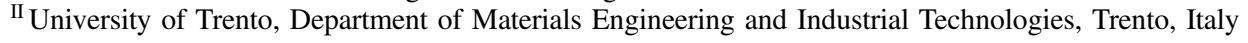

Received September 17, 2010; accepted October 1, 2010

Instrumental line profile / Profile aberrations / Synchrotron radiation XRPD / Debye-Scherrer geometry / Position sensitive detector / MYTHEN / TOPAS / PM2K / WPPM

Abstract. The main aberrations affecting data collected with 1D position sensitive detectors in Debye-Scherrer capillary geometry are examined, and analytical corrections proposed. The equations are implemented in two of the most advanced software based on the Rietveld and Whole Powder Pattern Modelling methods, respectively, for structure and microstructure analysis. Application to MYTHEN, a fast single photon counting detector developed at the Swiss Light Source, is discussed in detail.

\section{Introduction}

MYTHEN is a fast single photon counting 1D positionsensitive silicon microstrip detector developed at the Swiss Light Source (SLS) capable of acquiring X-ray Powder Diffraction patterns simultaneously over $120^{\circ}$ in $2 \theta$ [1-3]. Its design is modular, so that systems of different $2 \theta$ angular ranges can be assembled. The system installed at the SLS is made of 24 modules, each consisting of a silicon microstrip sensor with photon counting readout electronics for a total of 30720 independent channels working in parallel. Each sensor is made of $128050 \mu \mathrm{m}$ pitch strips, which are wire-bonded to the front-end electronics. The efficiency of the $\mathrm{Si}$ sensor is more than $85 \%$ for X-rays in the range $5-10 \mathrm{keV}$ and drops to about $25 \%$ at $20 \mathrm{keV}$ (limited by the thickness of the silicon wafer).

The readout electronics consists of 10 custom designed 128-channels ASICs per module operating in single photon counting mode, i.e. the charge created by the X-rays converting inside the silicon sensor collected by the closest strip is amplified and compared with a threshold. Only the signals higher than this reference are counted, so that all the electronic noise lower than the threshold is rejected and the statistics on the number of counts is purely Poisson-like.

\footnotetext{
* Correspondence author (e-mail: fabia.gozzo@psi.ch)
}

At the SLS, the detector modules are positioned at a distance of $760 \mathrm{~mm}$ from the sample and a He-filled box is used in order to limit the absorption and scattering of the diffracted photons due to air. Each module covers an angular range of $4.83^{\circ}$, with a gap of $0.17^{\circ}$ (42.5 channels) between two neighboring modules. Since the detector is mounted on one of the diffractometer arms, it is possible to move it in order to acquire patterns in different positions and merge the data sets to avoid the data loss in the gaps between the modules. The intrinsic angular resolution of the detector given by the strip pitch is approximately $0.004^{\circ}$, but the limit in this context is usually given by the size of the sample, which is projected onto the detector, as extensively discussed in this article.

The readout time of MYTHEN system is $250 \mu \mathrm{s}$ for 24-bit dynamic range down to $90 \mu$ s for 4-bit partial readout. The maximum frame rate (1 frame being 1 whole diffraction pattern) is, however, limited by the data transfer rate over the network and can be as much as 10-100 frames $\mathrm{s}^{-1}$ (24-4 bits) for the whole $120^{\circ}$ of the detector. Faster time-resolved measurements can, however, be performed by transferring data from a limited number of modules, thus obtaining a maximum frame rate of 100$900 \mathrm{~Hz}$ (24-4 bits) for a single module. In pump-andprobe experiments, on the other hand, the ns time scale can be achieved.

Thanks to the beamline optics fine tuning and the developed calibration procedures, the quality of the MYTHEN data in Debye-Scherrer transmission geometry is now comparable to that of traditional high resolution point detectors (in terms of FWHM resolution and peak profile shape), with the further advantage of fast and simultaneous acquisition of the full diffraction pattern [1-4].

MYTHEN is therefore optimal for time-resolved as well as high-resolution dose critical and in-situ non-ambient measurements. However, the lack of angular selection typical of a position sensitive detector, is a source of aberrations in the powder pattern in the form of peak displacement and profile modifications. These effects depend on various parameters, chiefly: sample size (e.g. capillary diameter), sample positioning, X-ray beam intensity distribution on the sample or powder distribution in the capillary, absorption, detector pixel size. 
All these aberrations are here analytically modelled for the case of a virtually aberration-free optics and fully parallel beam, as the Materials Science (MS) beamline at the SLS [4-6] can provide. The resulting expressions can be seamlessly incorporated in any data analysis program so as to be fully taken into account. We show here the implementation in TOPAS [7] and PM2K [8] based, respectively, on the Rietveld [9, 10] and Whole Powder Pattern Modelling [11-13] methods, together with their experimental validation.

\section{Instrumental contribution to peak profiles}

The observed powder diffraction peak profile $(h)$ of a polycrystalline material is broadened by both instrumental $(g)$ and specimen-related $(f)$ effects. Their combination is mathematically described by a convolution product [14]:

$$
h=g \otimes f .
$$

The instrumental and physical profiles are themselves the result of a convolution product of functions describing the individual sources of instrumental (e.g. optics, diffractometer, detector) and specimen-related (e.g. domain size, strain fields, dislocations, faulting) broadening [14, 15]:

$$
\begin{aligned}
& g=g_{1} \otimes g_{2} \otimes \ldots \otimes g_{n}, \\
& f=f_{1} \otimes f_{2} \otimes \ldots \otimes f_{m} .
\end{aligned}
$$

When we perform a microstructure analysis, independently of whether we apply traditional Line Profile Analysis (LPA) methods, e.g. based on the estimate of the integral breadth [12, 16, 17], or more advanced methods based on physical models of the microstructure [11-13], a careful characterization of the instrumental component of the observed line profile is mandatory, to safely extract the sample-related component from the observed profile.

The instrumental component can be either experimentally determined by recording a diffraction pattern on an appropriate standard under the same identical experimental conditions adopted for the analysis of the sample under investigation, or calculated from first principles starting from the instrument geometry (Fundamental Parameter Approach) [15, 18-22].

When measuring the instrumental contribution to the peak profile using a standard, the problem arises on what requirements the standard should meet. Ideally, it should provide a Dirac delta specimen-related broadening, condition that can be obtained with a large enough domain size not to contribute to the peak profile, but small enough to guarantee a sufficient particle statistics, absence of defects and a linear absorption coefficient comparable with that of the sample under investigation [15]. Being impossible to meet all these conditions (to some extent in contradiction with one another), the standard should be the closest approximation of the ideal one [23].

\section{Aberrations in Debye-Scherrer geometry}

In the following subsections, we describe the main aberrations and geometrical effects characterizing a position sen- sitive detector in Debye-Scherrer capillary geometry, and provide some experimental data purposely affected by those aberrations and effects. The following working conditions are assumed being met:

- aberration-free optics such as those available at the SLS-MS beamline [4-6]. Under these conditions the profile broadening due to the optics can be considered purely Gaussian [4];

- optimal calibration of the diffractometer and the detector [1];

- negligible absorption effects.

To limit the effects of the specimen holder, all powder samples were loaded in Lindemann glass no. 14 capillaries spun at approximately $10 \mathrm{~Hz}$.

\section{Specimen displacement correction}

In Debye-Scherrer (transmission) geometry, an offset of the capillary with respect to the goniometer centre causes a nonlinear shift in the $2 \theta$ positions that can be calculated as [24]:

$$
\Delta 2 \theta_{s d}=\arcsin \left(\frac{d_{L}}{R_{\mathrm{DS}}} \sin 2 \theta\right)-\arcsin \left(\frac{d_{V}}{R_{\mathrm{DS}}} \cos 2 \theta\right),
$$

where $d_{L}$ and $d_{V}$ denote, respectively, the displacement of the capillary in the direction of the beam and perpendicular to it, and $R_{\mathrm{DS}}$ is the sample-to-detector distance (see Fig. 1). For $1 \mathrm{D}$ microstrip detectors, the $R_{\mathrm{DS}}$ and the strip pitch $p$ (strip-to-strip distance) define the intrinsic resolution of the detector. For the MYTHEN mounted at the SLS-MS beamline, for instance, the intrinsic resolution is approximately $0.004 \mathrm{deg}$ in $2 \theta$, being $R_{\mathrm{DS}}=760 \mathrm{~mm}$ and $p=50 \mu \mathrm{m}[1]$.

Equation (3) was checked on XRPD data recorded on the NIST SRM 640c Silicon powder [25] loaded in a $0.3 \mathrm{~mm}$ capillary. Patterns were recorded at a photon energy of $15.5 \mathrm{keV}$ at nominal zero $d_{L}$ and $d_{V}$ (corresponding to a very careful positioning of the capillary in the rotation centre of the diffractometer) and, then, at several values of $d_{L}$ and $d_{V}$.

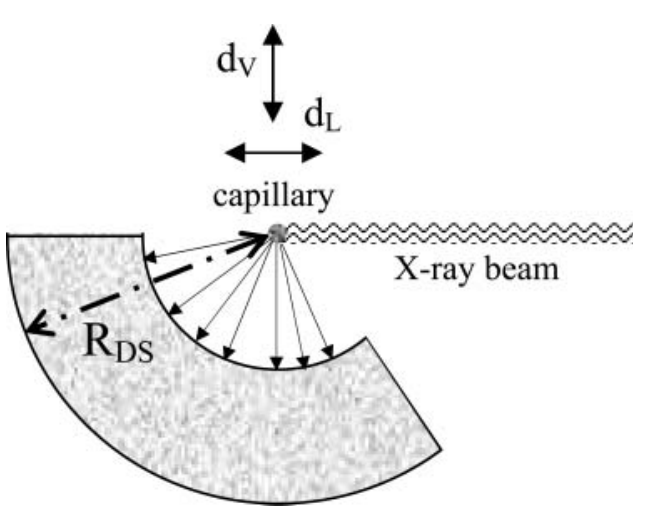

Fig. 1. Debye-Scherrer (transmission) experimental set up with MYTHEN detector: $d_{L}$ is the sample displacement along the direction of the beam, $d_{V}$ in the direction perpendicular to the beam and $R_{\mathrm{DS}}$ (760 $\mathrm{mm}$ at the SLS-MS powder diffraction beamline) is the sampleto-detector distance. 

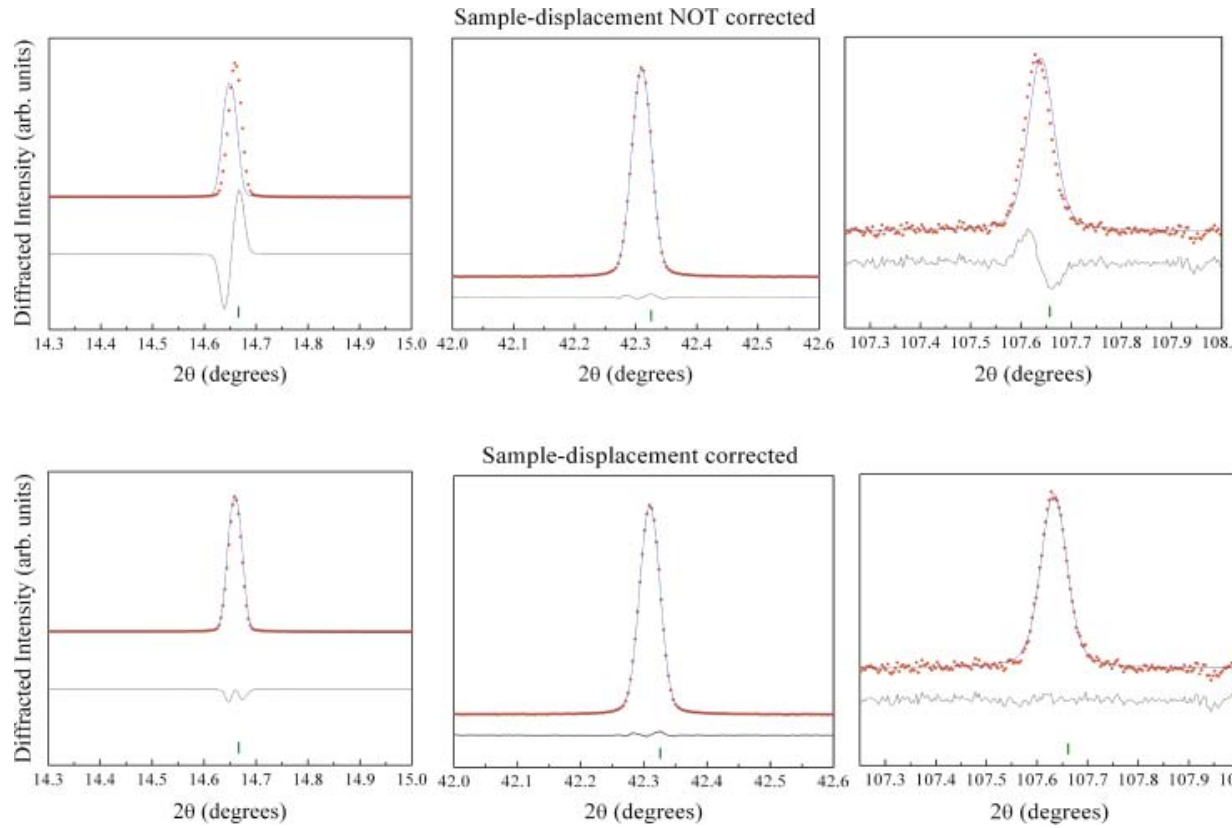

\section{Implementation in TOPAS}

The macro language of TOPAS allows an easy implementation of the specimen correction formula by the user (see Appendix A). The macro works on each peak, applying an offset to Bragg position, so the following is actually implemented:

$$
\begin{aligned}
\Delta 2 \theta_{h k l}= & \arcsin \left(\frac{d_{L}}{R_{\mathrm{DS}}} \sin 2 \theta_{h k l}\right) \\
& -\arcsin \left(\frac{d_{V}}{R_{\mathrm{DS}}} \cos 2 \theta_{h k l}\right)
\end{aligned}
$$

representing an excellent approximation for Eq. (3) in the case of narrow reflections. Figure 2 shows, as an example, the result of the whole pattern Pawley refinement [26] without (upper raw) and with (lower raw) the use of the sample displacement macro. For better clarity, only three Si reflections at low, medium and high $2 \theta$ angles are shown.

The Pawley refinements were performed with the following strategy. Appropriate profile shape parameters were identified via an empirical parameterization of the first few peaks of the pattern recorded at nominal zero $d_{L}$ and $d_{V}$. A pseudo-Voigt profile function was used for the fit, folded with a Gaussian that empirically describes the de-
Fig. 2. (colour online) Effect of sample-displacement correction on the whole powder Pawley refinement of NIST SRM 640C Si. Three Si reflections (111, 422 and 66̄) are shown for the data collected at nominal values $d_{L}=+0.3 \mathrm{~mm}$ and $d_{V}=0 \mathrm{~mm}$ (raw data: red-dots, fitting: blue-continuous line, residual difference: darkgrey-continuous line below). $R_{w p}$ and GoF improved from 2.31/1.52 obtained without correction (upper row) to $1.56 / 1.03$ obtained with the correction (lower row, see also Table 1).

Table 1. Results of Pawley refinements performed on diffraction patterns recorded at nominal zero $d_{V}$ displacement and variable $d_{L}$ values (see text for details).

\begin{tabular}{llcll}
\hline $\begin{array}{l}d_{L}, \\
\text { nominal } \\
(\mathrm{mm})\end{array}$ & $\begin{array}{l}d_{L, \text { fit }} / d_{V}, \text { fit } \\
\text { with sample displ. macro }\end{array}$ & $\begin{array}{l}2 \theta_{\text {zero-offset,fit }} \times 10^{-4} \mathrm{deg} \\
\text { with sample-displ. macro }\end{array}$ & $\begin{array}{l}R_{w p} / G o F \\
w / o \text { sample-displ. macro }\end{array}$ & $\begin{array}{l}R_{w p} / G o F \\
\text { with sample-displ. macro }\end{array}$ \\
\hline 0.0 & $-0.00(2) /-0.00(2)$ & $-10(10)$ & $1.56 / 1.03$ & $1.56 / 1.03$ \\
-0.3 & $-0.27(2) /-0.04(2)$ & $+30(10)$ & $2.29 / 1.50$ & $1.58 / 1.03$ \\
-0.2 & $-0.17(2) /-0.02(2)$ & $+20(10)$ & $1.84 / 1.22$ & $1.57 / 1.04$ \\
-0.1 & $-0.07(2) /-0.02(2)$ & $+20(10)$ & $1.63 / 1.08$ & $1.57 / 1.04$ \\
+0.1 & $+0.12(2) /-0.00(2)$ & $0(10)$ & $1.65 / 1.08$ & $1.58 / 1.04$ \\
+0.2 & $+0.22(2) /+0.00(2)$ & $0(10)$ & $1.89 / 1.24$ & $1.57 / 1.03$ \\
+0.3 & $+0.33(2) /+0.02(2)$ & $-20(10)$ & $2.31 / 1.52$ & $1.56 / 1.03$ \\
\hline
\end{tabular}

pendence of the peak FWHM on the capillary diameter (see next section) and with a top-hat function describing the pixel spread. The dependence of the profile FWHM on $2 \theta$, mainly due to the optics, was then successfully obtained using the whole diffraction pattern $\left(\max 2 \theta\right.$ of $\left.116^{\circ}\right)$ and the modified Thompson-Cox-Hastings pseudo-Voigt TCHZ with $U, V, W, X$ refineable parameters [4, 10].

The low-angle asymmetry due to the axial divergence was modelled using the Finger-Cox-Jephcoat [27] function with the two refineable parameters, s (capillary length) and $h$ (receiving slits length), set at the experimentally adopted values of $S=3 \mathrm{~mm}$ and $H=8 \mathrm{~mm}$. The parameters were then fixed to their convergence values (Gaussian_FWHM $=0.0163(3), \quad$ Hat_FWHM $=0.0315(3)$, $U=0.00013(3), \quad V=-0.00152(3), \quad W=0.00021(3)$, $X=0.01526(3), S=2.99(2), H=7.99(2))$ for all subsequent analyses of data recorded at different specimen displacement values. Only the $2 \theta$ zero offset was left free to vary, but its value never changed from one fit to another for more than one detector strip width (ca. $0.004 \mathrm{deg}$ in $2 \theta$ ).

The Pawley refinements of all data files with nominal zero $d_{V}$ and variable $d_{L}$ sample displacement show a remarkable quantitative agreement between nominal and refined values (Table 1). A weak correlation with $d_{V}$ is present, increasing with the induced $d_{L}$. 
The patterns corresponding to induced $\mathrm{d}_{\mathrm{V}}$ displacements did not show the expected peak shifts. The specimen displacement macro was in all cases improving the fit, although not quantitatively returning the nominal $d_{V}$ values, owing to the low precision of the $y$-motor (soon to be replaced with a more reliable, optically encoded one).

\section{Implementation in PM2K}

The Whole Powder Pattern Modelling (WPPM) [11-13] is based on Eqs. (1) and (2) for the generation of peak profiles. Each $\{h k l\}$ reflection family is split into its subcomponents $h k l$ that are individually modelled to fully account for defects influencing them in a peculiar way (e.g. stacking faults [13, 28]). Fully equivalent $h k l$ can be grouped and assigned a cumulative weight $w_{h k l}$. In reciprocal space, peak intensity then reads:

$$
I_{\{h k l\}}\left(d^{*}, d_{\{h k l\}}^{*}\right)=k\left(d^{*}\right) \cdot \sum_{h k l} w_{h k l} I_{h k l}\left(s-s_{h k l}\right) .
$$

The peak centroid is defined with respect to the Bragg position $\left(d_{\{h k l\}}^{*}=2 \sin \left(\theta_{\{h k l\}}\right) / \lambda\right)$ calculated on the basis of the given lattice. When needed, a displacement $\delta_{h k l}$ can be applied to each individual $h k l$ profile:

$$
s-s_{h k l}=d^{*}-\left(d_{\{h k l\}}^{*}-\delta_{h k l}\right) .
$$

It is important to note that, whenever possible, $\theta$ or $s$ dependent aberrations are not applied peakwise (i.e. considering just an average shift of the Bragg position), but pointwise. The differences can be appreciated on wide peaks or in the peak tails, a main concern for microstructure analysis.

The intensity of each $h k l$ peak is then calculated as a convolution of all broadening components. A simplification of the problem is obtained when all operations are done in Fourier space. In fact, the convolution theorem states that the Fourier transform of a convolution is equal to the product of the Fourier transforms of the functions to be folded. If $\boldsymbol{C}_{h k l}(L)$ is the product of all such functions defined in Fourier space for a profile subcomponent, then the intensity can be easily calculated as:

$$
I_{h k l}\left(s-s_{h k l}\right)=\int_{-\infty}^{\infty} \boldsymbol{C}_{h k l}(L) \exp \left(2 \pi i L \cdot\left(s-s_{h k l}\right)\right) \mathrm{d} L .
$$

It is worth noting that the Fourier space dual to reciprocal space enters Eq. (7). The availability of the Fourier transform of the broadening sources, including those relative to the instrumental contribution, allows their fast incorporation into the WPPM.

The capillary displacement has been introduced as a pointwise correction in the PM2K software [8] implementing the WPPM. The performance of PM2K is quite similar to that of TOPAS, in that the induced capillary shift is correctly retrieved from the experimental data. The actual values are slightly different, as a substantially different peak shape was employed in the analysis. All peaks were modelled using a pseudo-Voigt function (symmetrical in $s$ ) whose Full Width at Half Maximum (FWHM) and shape was parameterised according to a polynomial in $\tan (\theta)$ and in $\theta$, respectively (Caglioti plots [29]) described by the
Table 2. Results of WPPM refinements performed on diffraction patterns recorded at nominal zero $d_{V}$ displacement and variable $d_{L}$ values modelling all aberrations and using the specimen displacement correction (see text for details). Values are referred to the refined nominal zero.

\begin{tabular}{llcc}
\hline $\begin{array}{l}d_{L, \text { nominal }} \\
(\mathrm{mm})\end{array}$ & $\begin{array}{l}d_{L, \text { fit }} / d_{V, \text { fit }} \\
(\mathrm{mm})\end{array}$ & $\begin{array}{l}2 \theta_{\text {zero-offset, fit }} \\
\times 10^{-4} \mathrm{deg}\end{array}$ & $R_{w p} / G o F$ \\
\hline 0.0 & $-0.000(2) /-0.000(2)$ & $2(1)$ & $1.75 / 1.16$ \\
-0.3 & $-0.284(2) /-0.004(2)$ & $6(1)$ & $1.64 / 1.08$ \\
-0.2 & $-0.194(2) /-0.004(2)$ & $7(2)$ & $1.71 / 1.13$ \\
-0.1 & $-0.102(2) /-0.003(2)$ & $7(1)$ & $1.75 / 1.14$ \\
+0.1 & $+0.098(2) /-0.004(2)$ & $9(1)$ & $1.70 / 1.10$ \\
+0.2 & $+0.192(2) /+0.002(2)$ & $11(1)$ & $1.71 / 1.12$ \\
+0.3 & $+0.286(2) /+0.004(2)$ & $7(1)$ & $1.74 / 1.15$ \\
\hline
\end{tabular}

parameters $\quad W=1.087(6) \times 10^{-4}, \quad V=2.804(6) \times 10^{-3}$, $U=-2.667(2) \times 10^{-4}, \quad a=5.685(5) \times 10^{-2}, \quad b=$ $5.271(5) \times 10^{-3}$. As expected, the peaks have a very small Lorentz character. The pseudo-Voigt was folded with the functions defined below accounting for the change in profile shape and asymmetry due to the geometry.

All instrumental profile parameters were refined for each point, as to simulate what is usually done when modelling the instrumental contribution: the correct position with respect to the refined zero is recovered. The values are presented in Table 2 with respect to the modelled centroid, as done for the TOPAS data. Statistical indices are worse than in Table 1 due to a less accurate fitting of the background.

The trend of the Caglioti plots is almost indistinguishable in the various cases, witnessing the stability of the algorithms and of the implemented profile functions.

\section{Profile shape dependence on capillary diameter $D$ and cylindrical wobbling of radius $w$}

Each $h k l$ Bragg peak in a powder diffraction pattern collected from a capillary specimen of diameter $D$ perfectly aligned along the spinning axis (no wobbling of any type), is broadened by a certain profile. This arises because the capillary is not a point, therefore its scattering density within the diffraction plane (a uniform circle) must be projected onto the angular coordinates along the detector surface (the latter is assumed locally flat and orthogonal to the scattered beam). It results the following expression:

$$
f(x)=\frac{2}{\pi x_{0}^{2}} \sqrt{x_{0}^{2}-x^{2}}:-x_{0} \leq x \leq x_{0},
$$

where:

$$
\begin{aligned}
& x=2 \theta-2 \theta_{h k l}[\mathrm{deg}] \\
& x_{0} \equiv \frac{180}{\pi} \frac{D / 2}{R_{\mathrm{DS}}}=\frac{90 D}{\pi R_{\mathrm{DS}}} .
\end{aligned}
$$

The Fourier transform of $f(x)$ can be easily computed as:

$$
P(q)=\int_{-x_{0}}^{+x_{0}} f(x) \cos (2 \pi q x) \mathrm{d} x=\frac{J_{1}(2 K)}{K},
$$


where $J_{1}(x)$ is Bessel function of first kind and order 1, and $K=\pi q x_{0}$.

The accurate alignment of a capillary in the rotation centre of the goniometer head is quite difficult, and some residual wobbling is inevitably present. The simplest case is that of a capillary slightly displaced from the spinning axis (goniometer centre), describing a circular trajectory around it when spun. It is assumed that the capillary is always completely bathed by the X-ray beam during its spinning.

A capillary of diameter $D$ wobbling cylindrically around the spinning axis with a trajectory radius $w$, causes a profile broadening given by the following convolution product:

$$
\begin{aligned}
& f_{w}(x)=f(x) \otimes g_{w}(x)=\int_{-\infty}^{+\infty} f(t) g_{w}(x-t) \mathrm{d} t \\
& g_{w}(x)=\frac{1}{2 \pi} \int_{0}^{2 \pi} \delta\left(x-u_{0} \cos \phi\right) \mathrm{d} \phi \\
&=\frac{1}{\pi \sqrt{u_{0}^{2}-x^{2}}}:-u_{0} \leq x \leq u_{0}, \\
& u_{0} \equiv \frac{180}{\pi} \frac{w}{R_{\mathrm{DS}}}=x_{0} \frac{2 w}{D}
\end{aligned}
$$

where $g_{w}(x)$ describes the angular density of the projection of the wobbling capillary centre onto the detector surface (as before, assumed locally flat and orthogonal to the scattered beam). The factor 2 in (13) arises from the fact that $D$ is defined as a diameter whereas $w$ is a radius. The Fourier transform of $g_{w}(x)$ is easily calculated as:

$$
G_{w}(q)=\int_{-w_{0}}^{+w_{0}} g_{w}(x) \cos (2 \pi q x) \mathrm{d} x=J_{0}(2 K(2 w / D))
$$

and, therefore, the Fourier transform of (11) is:

$$
P_{w}(q)=P(q) \cdot G_{w}(q)=K^{-1} J_{1}(2 K) J_{0}(2 K(2 w / D)) .
$$

An alternative simplified approach could also be workable under certain circumstances. We can in fact replace $f(x)$ (Eq. (8)) over the interval $\left(-x_{0}, x_{0}\right)$ with an equivalent area Gaussian [1] whose standard deviation, expressed in deg, is:

$$
\sigma_{\text {cap }}=\left(\frac{45}{\pi}\right)\left(\frac{D}{R_{\mathrm{DS}}}\right) \text {. }
$$

Multiplying (16) by the factor $2 \sqrt{2 \ln 2}$ converts the standard deviation of the Gaussian into its FWHM that we can directly extract from a diffraction pattern.

The cylindrical wobbling discussed above can also be reasonably approximated, in the limit of small wobbling radius $(w \ll 0.25 D)$, with a Gaussian function of standard deviation [1]:

$$
\sigma_{\mathrm{wob}}=\frac{90 \sqrt{2}}{\pi} \frac{w}{R_{\mathrm{DS}}}=\sqrt{8} \frac{w}{D} \sigma_{\text {cap }} .
$$

For $w \geq 0.25 D$ (severe wobbling) the peak shape significantly changes (eventually splitting) and the Gaussian approximation no longer works. In such cases, the exact implementation of the profile function is necessary.

\section{Implementation in TOPAS}

The effectiveness of the Gaussian approximation in describing the aberrations due to the finite size of a slightly wobbling capillary under the hypothesis of $w \ll 0.25 D$, was checked against Pawley refinements of XRPD data recorded with a MYTHEN detector on the NIST SRM 640C Silicon powder [25], loaded in $0.2,0.3,0.4,0.5$, $0.8 \mathrm{~mm}$ capillaries.

A photon energy of $28 \mathrm{keV}$ was employed to minimize the effects of absorption for the thicker capillaries. Slightly higher $R_{w p}$ were, in general, obtained for this set of refinements compared with the $15.5 \mathrm{keV}$ data discussed before. This is quite common for SLS-MS data, as the peak profile shape at photon energies $>20 \mathrm{keV}$ is intrinsically more difficult to model (the optics start playing a role in the description of the peak profile).

Following the same refinement strategy described in the previous section for the evaluation of the sample displacement correction, a satisfactory Pawley refinement $\left(R_{w p}=4.50 ; G o F=1.04\right)$ was first achieved for the $0.2 \mathrm{~mm}$ capillary data by including a Gaussian convolution function with a FWHM fixed at the theoretical expected value of $8.95 \mathrm{mdeg}$, predicted by Eq. (16). Then, the XRPD patterns recorded at the other $D$ values were analysed by fixing the instrumental parameters to the values refined on the $D=0.2 \mathrm{~mm}$ XRPD data, and refining only the Gaussian_FWHM.

Table 3 shows the results of this series of Pawley refinements and Fig. 3 the corresponding plots.

We can see that up to ca. $D=0.4 \mathrm{~mm}$ the simple Gaussian approximation for the peak profile broadening due to finite capillary diameter and moderate residual wobbling works reasonably well. Above this value, the returned value of the Gaussian_FWHM contribution is systematically underestimated. The graphical inspection of the corresponding Pawley refinements confirms that the peak FWHM is underestimated because the peaks split and the actual shape is no longer modelled by an appropriate function. In such cases, Eq. (11) should be employed.

Table 3. Results of Pawley refinements performed on diffraction patterns recorded at increasing capillary diameter $D$. All refinement parameters, but the $2 \theta$ zero offset, were fixed to the convergence values obtained from the $D=0.2 \mathrm{~mm}$ capillary dataset.

\begin{tabular}{lllll}
\hline $\begin{array}{l}D \\
(\mathrm{~mm})\end{array}$ & $\begin{array}{l}\sigma_{\text {cap }} \\
\text { (mdeg) } \\
\text { calculated }\end{array}$ & $\begin{array}{l}\text { Gaussian } \\
\text { FWHM } \\
(\text { mdeg) } \\
(2 \sqrt{\ln 2})\end{array}$ & $\begin{array}{l}\text { Gaussian } \\
\text { FWHM } \\
\text { (mdeg) } \\
\text { fit }\end{array}$ & $R_{w p} / G o F$ \\
\hline 0.2 & 3.8 & 8.95 & 9 & $4.50 / 1.04$ \\
0.3 & 5.7 & 13.42 & $16.9(3)$ & $4.61 / 1.11$ \\
0.4 & 7.5 & 17.66 & $26.1(3)$ & $5.36 / 1.39$ \\
0.5 & 9.4 & 22.13 & $18.6(3)$ & $5.33 / 1.41$ \\
0.8 & 15.1 & 35.56 & $31.6(3)$ & $7.85 / 2.56$ \\
\hline
\end{tabular}


Fig. 3. (colour online) Pawley refinement of NIST SRM 640C patterns recorded on capillaries of increasing diameter $D$. The cases $D=0.3 \mathrm{~mm}$ (a) and $D=0.4 \mathrm{~mm}$ (b) are shown (raw data: red-dots, fitting: blue-continuous line, residual difference: dark-grey-continuous line below). The profile dependence on $D$ was modelled with a simple Gaussian component as discussed in the text.
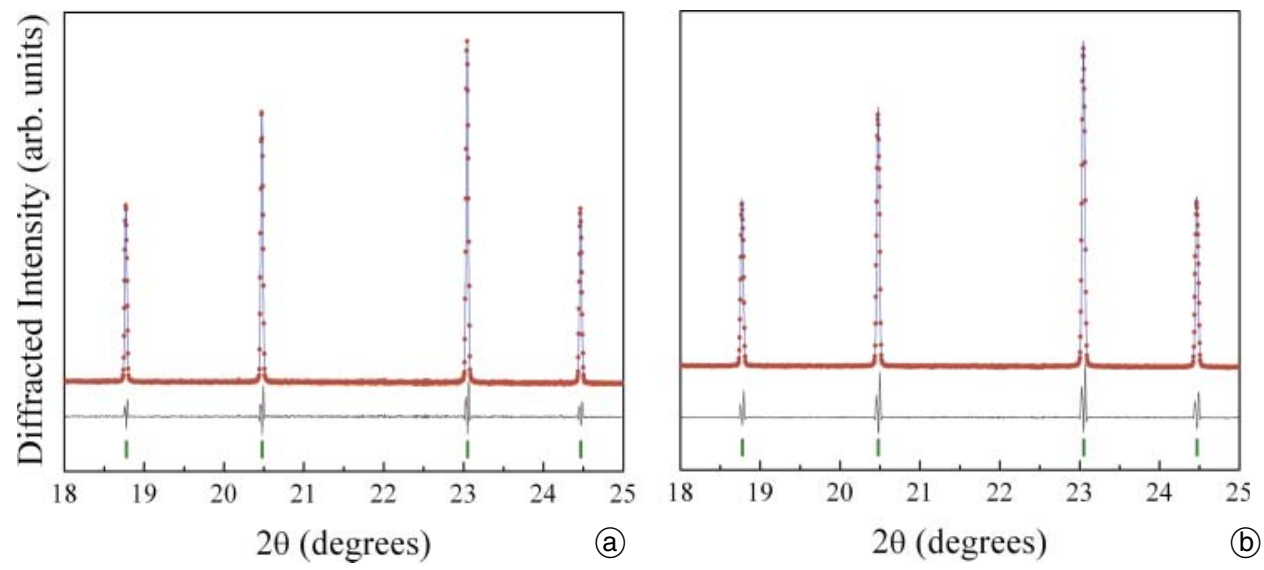

\section{Implementation in PM2K}

The nature of the WPPM equations calls for a direct implementation of the analytical formulae proposed above, thus avoiding the limitations of the approximate corrections. Two possible options are viable: $(i)$ working with the function defined in $2 \theta$ or (ii) employing the analytical Fourier transform. All Fourier transforms proposed above, however, have been obtained as a result of the direct transformation from the $2 \theta$ space to its dual $q$ as defined in Eq. (10) that, as a matter of fact, has no direct physical interpretation. The transformation from reciprocal space to correlation length space (i.e. from $s$ to $L$ ) is the one physically meaningful for the WPPM. This type of transformation is quite difficult to perform analytically without approximations. By suitably expanding the running variable $s-s_{h k l}$ as:

$$
\begin{aligned}
s-s_{h k l} & =\frac{2\left[\sin \left(\gamma\left(x+2 \theta_{h k l}\right)\right)-\sin \left(\gamma 2 \theta_{h k l}\right)\right]}{\lambda} \\
& =x \frac{2 \gamma}{\lambda} \cos \left(\gamma 2 \theta_{h k l}\right)-x^{2} \frac{\gamma^{2}}{\lambda} \sin \left(\gamma 2 \theta_{h k l}\right)+O\left(x^{3}\right),
\end{aligned}
$$

where $\gamma=1 / 2$ for radians and $\gamma=\pi / 360$ for degrees, and taking just a first order approximation, we have:

$$
s-s_{h k l} \approx \alpha x, \quad \alpha=\frac{2 \gamma}{\lambda} \cos \left(\gamma 2 \theta_{h k l}\right) .
$$

As this change of variable implies just the multiplication by a constant, and being the Fourier transform a linear operator, we can obtain the transform from $s$ to $L$ from the $2 \theta$ to $q$ one (proposed above) simply by replacing $q$ with $\alpha L$. For instance, Eq. (10) modifies into:

$$
P(L) \approx \int_{-x_{0}}^{+x_{0}} f(x) \cos (2 \pi \alpha L x) \mathrm{d} x=\frac{J_{1}\left(2 \pi x_{0} \alpha L\right)}{\pi x_{0} \alpha L} .
$$

The error made by ignoring the other terms in the expansion is expected to be of the order of magnitude of the ratio between the second and first order in the expansion i.e.:

$$
r=-x \frac{\gamma}{2} \tan \left(\gamma 2 \theta_{h k l}\right)
$$

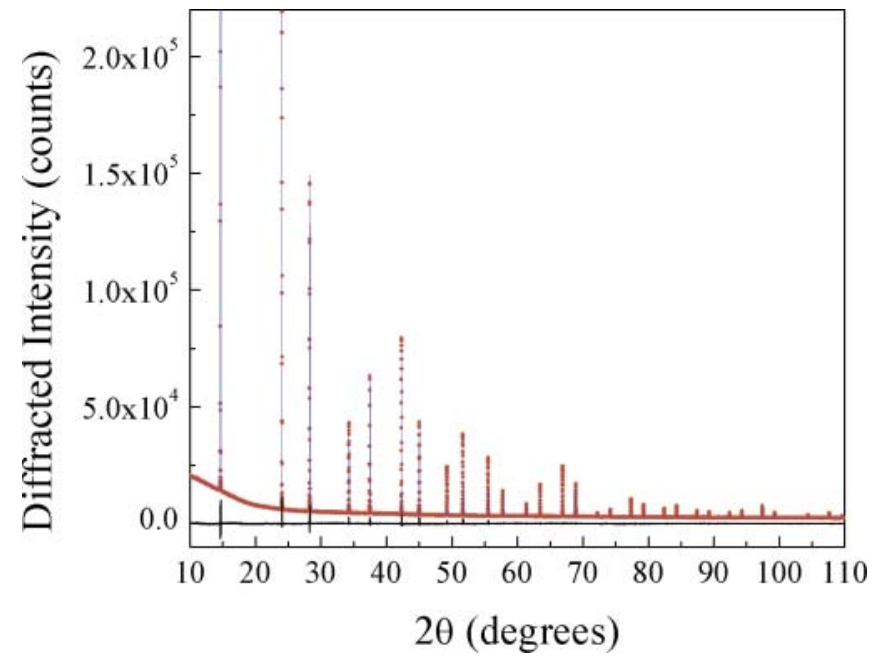

Fig. 4. (colour online) PM $2 \mathrm{~K}$ modelling of the $\mathrm{Si}$ data $D=0.3 \mathrm{~mm}$, $d_{L}=+0.3 \mathrm{~mm}, d_{V}=0.0 \mathrm{~mm}$ (raw data: red-dots, fitting: blue-continuous line, residual difference: dark-grey-continuous line below).

Even for a large capillary size $(1 \mathrm{~mm})$, the absolute value of $r$ is below $2 \times 10^{-3}$ for angles as large as $160^{\circ}$ in $2 \theta$.

Figure 4 shows the PM2K modelling result of the $\mathrm{Si}$ dataset previously analysed with TOPAS and shown in Fig. $2\left(D=0.3 \mathrm{~mm}, d_{L}=+0.3 \mathrm{~mm}, d_{V}=0\right)$. The detail of the (220) profile shape, with a separation of the various

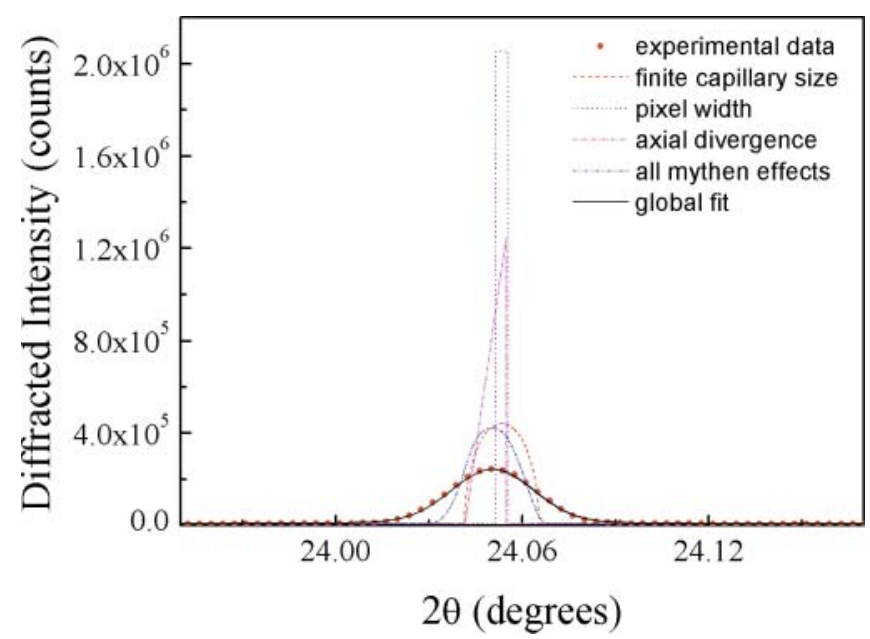

Fig. 5. Detail of the various broadening components employed by PM2K to model the (220) Si peak of the pattern of Fig. 4. 


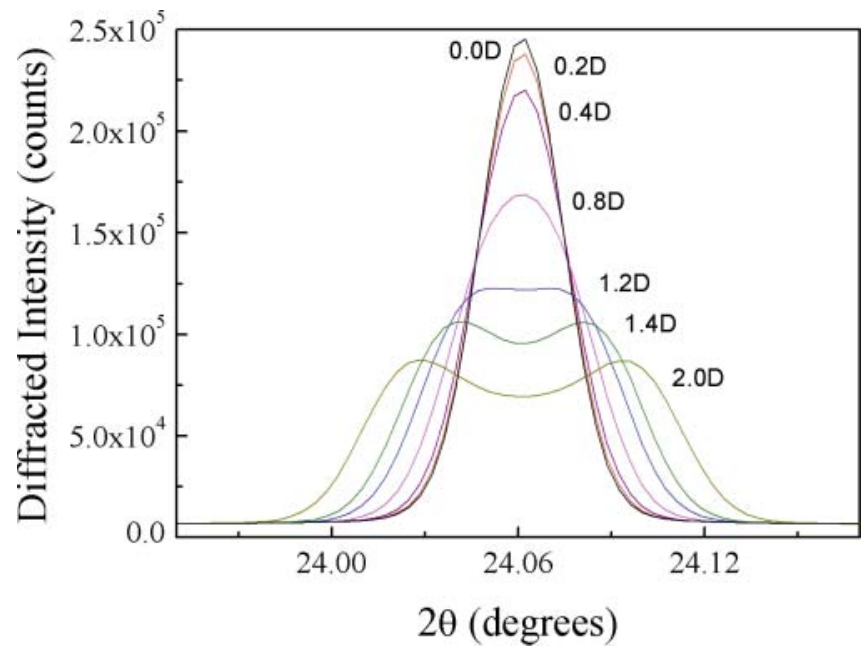

Fig. 6. Effect of wobbling on the (220) Si peak. The intrinsic profile modelled on the experimental data $(D=0.3 \mathrm{~mm}, w=0.0 D$, see Figs. 4 and 5) is superimposed to simulations performed by adding wobbling effects with increasing $w$ (referred to the capillary diameter $D$ of $0.3 \mathrm{~mm}$ ) to the refined data.

components contributing to the final profile is shown in Fig. 5.

In particular, Fig. 5 shows the various contributions to the profile broadening of pixel width size, axial divergence, finite capillary size, and their combination is provided together with the result of the pattern modelling. It can be appreciated that the major broadening contribution is given by the finite capillary size, whereas the only source of peak shift - considering a perfectly centred capillary - is the axial divergence.

Figure 6 shows the modification that occurs to the peak profile when applying the wobbling correction. The peak progressively broadens, with a clear splitting of the peak observed for large values of $w$.

As the splitting is dependent on $2 \theta$ and is small for small wobbling radius, it can be easily misinterpreted as anisotropic peak broadening if not properly taken into account.

\section{Further contributions to the profile}

Besides the effects related to the specimen holder, a set of further contributions need to be considered when modelling the data from 1D position sensitive detectors such as the MYTHEN, the major being certainly axial divergence and point spread function. Axial divergence is caused by the finite extension of illuminated specimen area and of the detector pixel perpendicular to the equatorial (beam) plane. In the set-up shown in this work (Fig. 1) axial divergence is not contrasted by any optics, as it happens for other geometries (e.g., Soller slits in the standard powder diffractometers).

The pixel spread function accounts for the discrete size of each of the counters in the detector. While further details can be found in the recent literature [1], a quick account is given here, as both contributions are considered in the modelling of the patterns shown here.

The axial divergence is well described by the function proposed by Finger, Cox and Jephcoat [27] and reprised by Prince and Toby [30]. Both a shift in the peak posi-

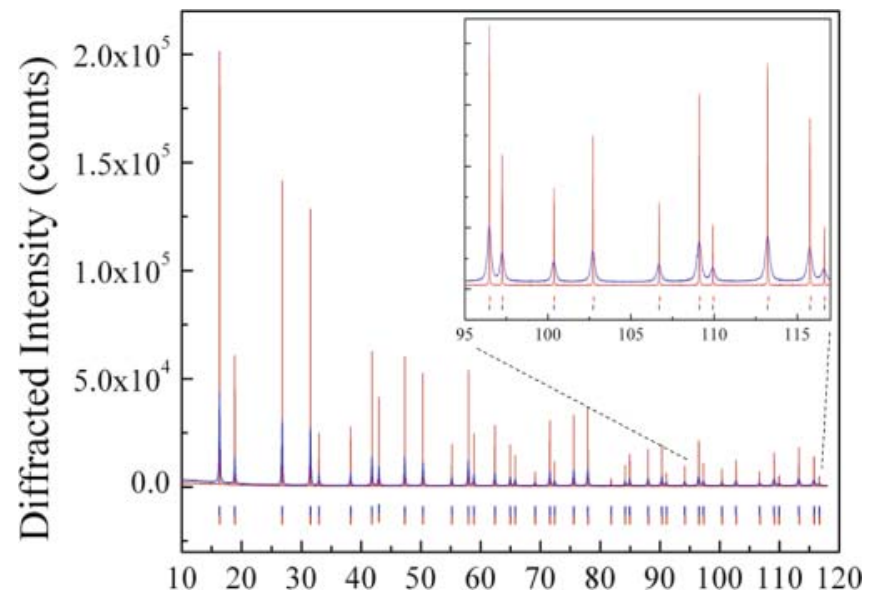

(a)

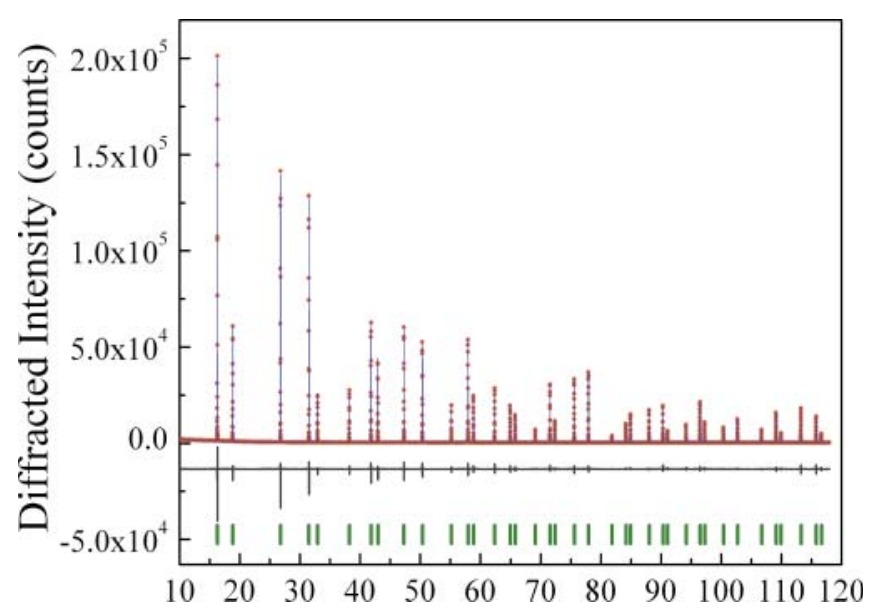

(b)

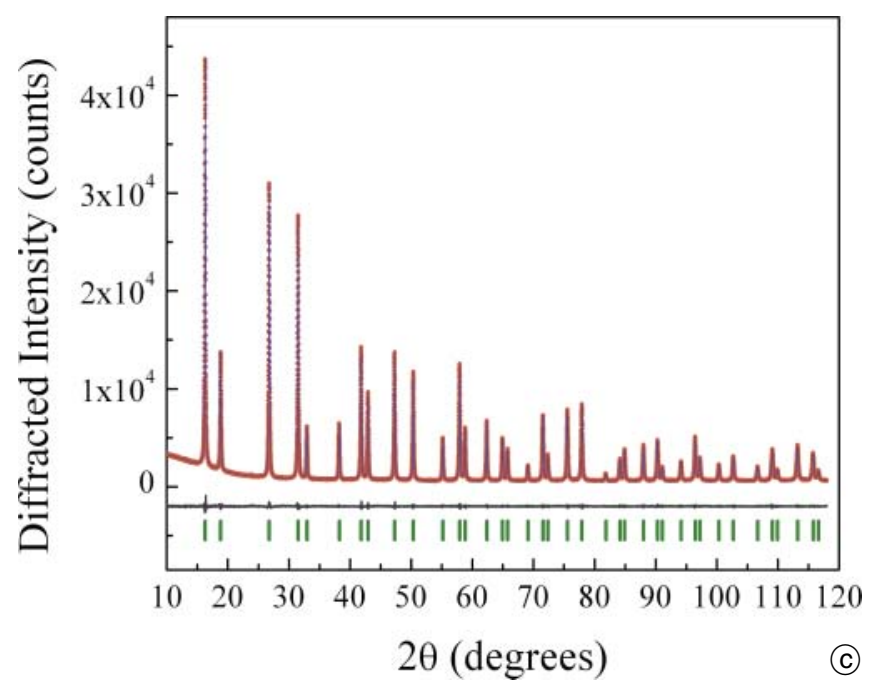

Fig. 7. (colour online) XRPD patterns of crystalline and nanocrystalline $\mathrm{CeO}_{2}$ (ceria) powders recorded with MYTHEN. Comparison between the patterns ((a): red curve (ceria-sharp), blue curve (ceriabroad)) and refinement results for the reference (b) and for the nanocrystalline (c) powders. Middle and bottom plots: raw data are reddots, fitting is the blue-continuous line, residual difference is the dark-grey-continuous line below). 
tions and an asymmetry are expected to be present, the sign of the latter showing a turning point at $2 \theta=90^{\circ}$.

The broadening arising from the finite pixel size can be well approximated by a unit area top-hat function, whose full width $\varepsilon$ is:

$$
\varepsilon=\frac{180}{\pi} \frac{p}{R_{\mathrm{SD}}}
$$

being $p$ the detector strip pitch (50 $\mu \mathrm{m}$ for the MYTHEN).

The Fourier transform of such a function is

$$
P_{\varepsilon}(q)=\int_{-\varepsilon / 2}^{\varepsilon / 2} \frac{1}{\varepsilon} \cos (2 \pi q x) \mathrm{d} x=\operatorname{sinc}(\pi q \varepsilon) .
$$

Both functions are already available as a subset of functions implemented in TOPAS and in the latest version of PM2K.

\section{A case of study: size-strain round robin specimen}

As an example of the performance that can be achieved by using the MYTHEN detector and the provided aberration correction formulae, we propose the analysis of the nanocrystalline $\mathrm{CeO}_{2}$ specimen employed for the sizestrain round robin conducted in 2004 by the Commission on Powder Diffraction of the International Union of Crystallography [31]. Data from a coarse grained specimen were provided to account for instrumental effects. Although this is not a certified profile standard, we used it to be consistent with the proposed case of study.

Tutorial input files are provided as part of the TOPAS software to analyse the original round robin data. The same tutorial will be followed here, starting from the naming convention: the nanocrystalline and reference $\mathrm{CeO}_{2}$ powders will be denoted as broad and sharp, respectively.

We recorded XRPD patterns of the 2004 round robin powders using MYTHEN and followed the TOPAS tutorial, based on the use of the effective double-Voigt approach [32]. Figure 7 shows the XRPD patterns recorded for 2 and $8 \mathrm{~s}$, on ceria-sharp and broad (a) and their corresponding Pawley refinements (b and $c$ ).

Empirical parameterization of the line profile shape of ceria-sharp was performed to carefully characterize the instrumental contribution to the line profile. The refined parameters were then fixed in the subsequent analysis of the nanocrystalline ceria powder to unfold the instrumental contribution to the line profile in the microstrain analysis.

Table 4 shows the results of the microstructure analysis generated by TOPAS using MYTHEN data compared with the published results using the round robin data.

Table 4. Direct comparison of the size-strain parameters (evaluated in TOPAS following the Double-Voigt Approach [32]) extracted from the microstructure analysis performed with TOPAS using the 2004 round robin data [31] and MYTHEN data.

\begin{tabular}{lll}
\hline & $\begin{array}{l}\text { 2004 round robin } \\
\text { XRPD data }\end{array}$ & $\begin{array}{l}\text { MYTHEN XRPD } \\
\text { data }\end{array}$ \\
\hline LVol-IB & $23 \pm 1 \mathrm{~nm}$ & $22.9 \mathrm{~nm}$ \\
$e_{0}$ & $0.007 \pm 0.003$ & 0.005 \\
\hline
\end{tabular}

MYTHEN XRPD patterns brought to a fairly good quantitative agreement with the round-robin results.

\section{Conclusions}

The major aberrations affecting data collected with state of the art $1 \mathrm{D}$ position sensitive detectors in Debye-Scherrer geometry have been presented and described analytically. The shift due to capillary misalignment and the aberrations coming from the finite size of the capillary, the presence of a wobbling, axial divergence and finite pixel size are fully described and implemented in the Rietveld method (TOPAS software) and in the Whole Powder Pattern Modelling (PM2K software). The quality of the corrected data allows the potential of a modern detector such as the MYTHEN developed at the Swiss Light Source, to be fully exploited. In this way structure and microstructure analysis can be seamlessly performed on patterns collected in fractions of seconds.

Acknowledgments. The experimental tests were performed at the Swiss Light Source, Paul Scherrer Institut. The authors would like to thank D. Meister and M. Lange for the competent technical support at the beamline, Davor Balzar for providing the ceria powders, Bernd Hinrichsen, Alan Coehlo and Arnt Kern for the kind and constant support in exploring TOPAS capabilities.

\section{Appendix - TOPAS macro}

The macro for the capillary displacement can be easily implemented in TOPAS as:

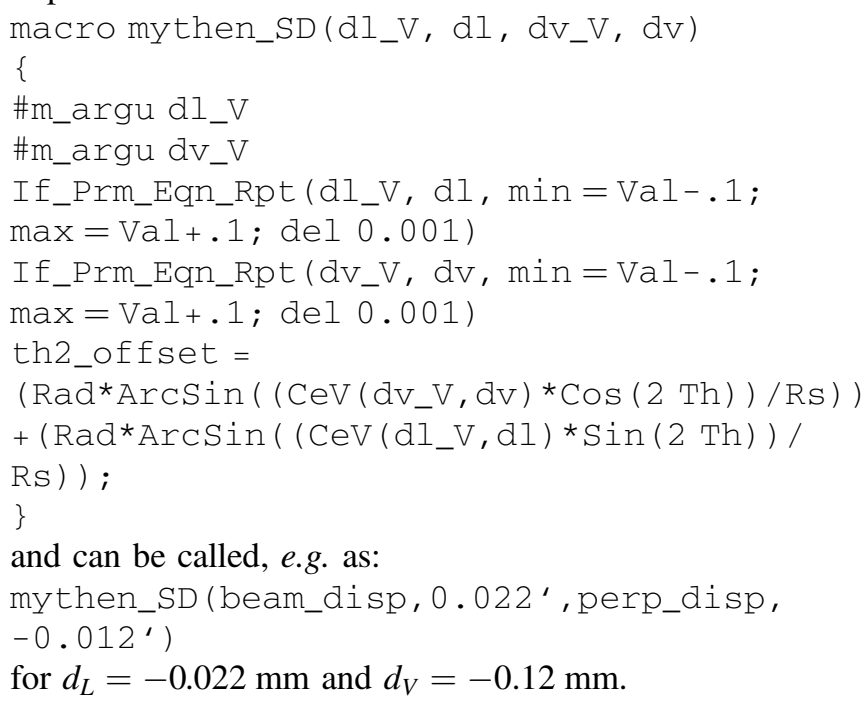

\section{References}

[1] Bergamaschi, A.; Cervellino, A.; Dinapoli, R.; Gozzo, F.; Henrich, B.; Johnson, I.; Kraft, P.; Mozzanica, A.; Schmitt, B.; Shi, X.: The MYTHEN detector for X-ray powder diffraction experiments at the Swiss Light Source. J. Synchrotron Rad. 17 (2010) 653-668.

[2] Bergamaschi, A.; Cervellino, A.; Dinapoli, R.; Gozzo, F.; Henrich, B.; Johnson, I.; Kraft, P.; Mozzanica, A.; Schmitt, B.: Photon counting microstrip detector for time resolved powder diffraction experiments, Nucl. Instrum. Methods Phys. Res. A604 (2009) 136-139.

[3] Bergamaschi, A.; Broennimann, C.; Dinapoli, R.; Eikenberry, E.; Gozzo, F.; Henrich, B.; Kobas, M.; Kraft, P.; Patterson, B.; Schmitt, B.: Performance of a single photon counting microstrip detector for strip pitches down to $10 \mu \mathrm{m}$, Nucl. Instrum. Methods Phys. Res. A591 (2008) 163-166. 
[4] Gozzo, F.; De Caro, L.; Giannini, C.; Guagliardi, A.; Schmitt, B.; Prodi, A.: The instrumental resolution function of synchrotron radiation powder diffractometers in the presence of focusing optics. J. Appl. Cryst. 39 (2006) 347-357.

[5] Patterson, B. D.; Abela, R.; Auderset, H.; Chen, Q.; Fauth, F.; Gozzo, F.; Ingold, G.; Kuehne, H.; Lange, M.; Maden, D.; Meister, D.; Pattison, P.; Schmidt, Th.; Schmitt, B.; Schulze-Briese, C.; Shi, M.; Stampanoni, M.; Willmott, P. R.: The materials science beamline at the Swiss Light Source: design and realization. Nucl. Instrum. Methods Phys. Res. Sect A540 (2005) 42-67.

[6] Gozzo, F.; Schmitt, B; Bortolamedi, Th.; Giannini, C.; Guagliardi, A.; Lange, M.; Meister, D.; Maden, D.; Willmott, P.; Patterson, B. D.: First Experiments at the Swiss Light Source Materials Science Beamline Powder Diffractometer. J. Alloys Compd. 362 (2004) 206-217.

[7] Bruker AXS: TOPAS V4: General profile and structure analysis software for powder diffraction data. - Users Manual, Bruker AXS, Karlsruhe, Germany (2008).

[8] Leoni, M.; Confente, T.; Scardi, P.: PM2K: A flexible program implementing Whole Powder Pattern Modelling, Z. Kristallogr. Suppl. 23 (2006) 249-254. The PM2K software is available free of charge for non-proprietary applications and non-profit institutions.

[9] Rietveld, H.: A profile refinement method for nuclear and magnetic structures. J. Appl. Cryst. 2 (1969) 65-71.

[10] Young, R. A.: The Rietveld Method, edited by R. A. Young, IUCr Book Series, Oxford University Press (1993).

[11] Scardi, P.; Leoni, M.; Dong, Y. H.: Whole diffraction patternfitting of polycrystalline fcc materials based on microstructure. European Physical Journal B18 [1] (2000) 23-30.

[12] Scardi, P.; Leoni, M.: Line profile analysis: pattern modelling versus profile fitting. J. Appl. Cryst. 39 (2006) 24-31.

[13] Scardi, P.; Leoni, M.: Whole Powder Pattern Modelling Acta Crystall. A58 (2002) 190-200.

[14] Klug, H. P.; Alexander, L. A.: X-Ray Diffraction Procedures: For Polycrystalline And Amorphous Materials, 2nd Edition. John Wiley \& Sons, New York (1974).

[15] Kern, A.; Coelho, A. A.; Cheary, R. W.: Convolution Based Profile Fitting, in Diffraction Analysis of the Microstructure of Materials, edited by E. J. Mittemeijer; P. Scardi (Berlin: Springer), 2004, pp. 17-49.

[16] Snyder, R. L.; Fiala, J.; Bunge, H. J.: Defect and microstructure analysis by diffraction, IUCr Monographs on Crystallography 10, Oxford University Press, New York (1999).

[17] Scardi, P.; Leoni, M.; Delhez, R.: Line broadening analysis using integral breadth methods: a critical review. J. Appl. Cryst. 37 (2004) 381-390.

[18] Cheary, R. W.; Coelho, A. A.: A fundamental parameters ap- proach to X-ray line-profile fitting, J. Appl. Cryst. 25 (1992) $109-121$

[19] Cheary, R. W. \& Coelho, A. A.: Synthesizing and fitting linear position-sensitive detector step-scanned line profiles, J. Appl. Cryst. 27 (1994) 673-681.

[20] Cheary, R. W.; Coelho, A. A.: Axial Divergence in a Conventional X-ray Powder Diffractometer. I. Theoretical Foundations, J. Appl. Cryst. 31 (1998) 851-861.

[21] Cheary, R. W.; Coelho, A. A.: Axial Divergence in a Conventional X-ray Powder Diffractometer. II. Realization and Evaluation in a Fundamental-Parameter Profile Fitting Procedure, J. Appl. Cryst. 31 (1998) 862-868.

[22] Zuev, A. D.: Calculation of the instrumental function in X-ray powder diffraction, J. Appl. Cryst. 39 (2006) 304-314.

[23] van Berkum, J. G. M.; Sprong, G. J. M.; de Keijser, Th. H.; Delhez, R.; Sonneveld, E. J.: The optimum standard specimen for X-ray diffraction line-profile analysis, Powder Diffr. 10 (1995) 129-139.

[24] McCusker, L. B.; Von Dreele, R. B.; Cox, D. E.; Louer, D.; Scardi, P.: Rietveld refinement guidelines. J. Appl. Cryst. 32 (1999) 36-50.

[25] Cline, J. P.; Deslattes, R. D.; Staudenmann, J.-L.; Kessler, E. G.; Hudson, L. T.; Henins, A.; Cheary R. W.: Certificate SRM 640c. (NIST Gaithersburg, MD 2000).

[26] Pawley, G. S.: Unit-cell refinement from powder diffraction scans. J. Appl. Cryst. 14 (1981) 357-361.

[27] Finger, L.W.; Cox, D. E; Jephcoat, A. P.: A correction for powder diffraction peak asymmetry due to axial divergence. J. Appl. Cryst. 27 (1994) 892-900. [25]

[28] Estevez-Rams, E.; Leoni, M.; Scardi, P.; Aragon-Fernandez, B.; Fuess, H.: On the powder diffraction pattern of crystals with stacking faults, Phil. Mag. 83 [36] (2003) 4045-4057.

[29] Caglioti, G.; Paoletti, A.; Ricci, F. P.: Choice of collimators for a crystal spectrometer for neutron diffraction, Nucl. Instr. 3 (1958) 223-228.

[30] Prince E., Toby B. H.: A comparison of methods for modeling the effect of axial divergence in powder diffraction. J. Appl. Cryst. 38 (2005) 804-807.

[31] Balzar, D.; Audebrand, N.; Daymond, M. R.; Fitch, A.; Hewat, A.; Langford, J. I.; Le Bail, A.; Louer, D.; Masson, O.; McCowan, C. N.; Popa, N.C.; Stephens, P. W.; Toby, B. H.: Sizestrain line-broadening analysis of the ceria round-robin sample. J. Appl. Cryst. 37 (2004) 911-924.

[32] Balzar, D.: Voigt-function model in diffraction line-broadening analysis in Microstructure Analysis from Diffraction, edited by R. L. Snyder, H. J. Bunge, and J. Fiala, International Union of Crystallography (1999). 